Fecha de recepción: diciembre 2017 Fecha de aceptación: marzo 2018 Versión final: julio 2019

\section{El lujo tecnológico. Distinción y esnobismo en un contexto de prohibición: el consumo de Apple en Argentina (2015-2018)}

María Eugenia Correa ${ }^{\star}$ y Matías J. Romani **

Resumen: El presente trabajo pretende discutir el alcance de la categoría de "distinción" y su aplicación problemática al mundo de la tecnología digital. Para ello toma como universo de análisis a los consumidores de Apple en la Argentina durante el último trienio (20151018), con el fin de comprender el impacto que impuso una circulación restringida de ciertos bienes sobre los valores corporativos que caracterizan a la marca. La hipótesis que se pretende demostrar es que recién a partir del 2010 -cuando se endurecieron las políticas en materia de importación de bienes tecnológicos- que la experiencia de consumo de Apple comenzó a percibirse como una estrategia de distinción entre los consumidores argentinos.

Palabras claves: Distinción - Dispositivos tecnológicos - Experiencias de consumo - Cultura material - Nuevo lujo - Status social - Esnobismo.

[Resúmenes en inglés y portugués en las páginas 204-205]

${ }^{(*)}$ Doctora en Ciencias Sociales (UBA). Magíster en Sociología de la Cultura y Análisis cultural (IDAES-UNSAM). Licenciada en Sociología (UBA). Investigadora adjunta del CONICET en el Instituto de Investigaciones Gino Germani, Facultad de Ciencias Sociales, UBA. Docente de grado y posgrado de esta misma Facultad. Correo: eugecorrea@yahoo.com.ar

${ }^{(*)}$ Licenciado en Sociología y candidato a Magíster en Comunicación y Cultura por la Universidad de Buenos Aires. Profesor de grado en el Ciclo Básico Común (UBA) y de posgrado en el Doctorado de la Universidad de Palermo (UP), en la especialización Cultura y Comunicación de FLACSO y en la Maestría de Derechos Humanos de la UNTREF. Investigador categorizado del Instituto de Investigaciones Gino Germani (IIGG - UBA) en el área Cultura y Sociedad. Correo: romani@cbc.uba.ar

\title{
Introducción
}

El presente trabajo pretende discutir el alcance de la categoría de "distinción" y su aplicación problemática al mundo de la tecnología digital y de la electrónica de consumo en particular. Para ello toma como universo de análisis a los consumidores de Apple en la 
Argentina durante el último trienio (2015-1018), con el fin de comprender el impacto que impuso una circulación restringida de ciertos bienes tecnológicos sobre los valores corporativos que caracterizan a esta marca global. Si bien es una constante que la marca Apple está asociada con el lanzamiento de productos de calidad, con un fuerte componente en materia de innovación y en diseño, la impronta de exclusividad con la que se la asocia en el resto del mundo está vinculada, mucho más con el modelo de negocios que propugna, que con el rasgo de escasez relativa con la que se distingue en la Argentina. A tal punto que la serie de estos productos contrasta con otras alternativas, no siempre más económicas, pero en general más accesibles desde el punto de vista de la distribución, por ejemplo, en términos del soporte técnico, promociones bancarias, canales de retail, etc. y con una experiencia de consumo mucho más orientada hacia el aspecto funcional y utilitario del producto.

La hipótesis que se pretende demostrar es que si bien la marca Apple tiene una larga presencia en la Argentina, que puede rastrearse hasta los años ochenta del siglo pasado, es recién a partir del 2010 -cuando se endurecieron las políticas en materia de importación de bienes tecnológicos- que la experiencia de consumo de Apple comenzó a percibirse como una estrategia de distinción entre los consumidores tecnológicos de Argentina. No sólo debido al incremento sustancial del valor comercial en el mercado doméstico, sino también a un correlativo aumento de su valor "social" en términos de status. De ahí que la posesión de un iPad, un iPhone o una Macbook, objetos que predominan entre las fracciones superiores de la clase media metropolitana, operan como un signo de distinción dentro del consumo tecnológico nacional, en la medida en que su condición particular de escasez relativa conecta con otras formas de la construcción de la identidad de la nueva clase media metropolitana como es el acceso al turismo en el exterior.

En términos empíricos, este artículo busca recuperar la mirada de los propios consumidores de la marca, sus percepciones y representaciones no sólo sobre las mercancías tecnológicas en general, sino también acerca de la experiencia del consumo de Apple en la Argentina buscando el contraste con los valores culturales que la marca promueve a nivel global. Si se toma como punto de partida la necesidad de analizar los fundamentos de la cultura material, el objetivo propuesto implica reconstruir el relato social de un objeto tecnológico que, bajo determinadas circunstancias, se convierte en una mercancía distintiva en cuanto su sentido va variando a lo largo del tiempo. De aquí se derivan algunos interrogantes: ¿cómo opera en los consumidores tecnológicos y específicamente, sobre estas elecciones de compra, el concepto de distinción? ¿Cómo se articula la existencia de Apple como mercancía exclusiva frente a la multiplicación de los dispositivos tecnológicos? ¿Cuál es la relación entre la adquisición de un producto Apple y el turismo en el exterior? ¿Puede establecerse una conexión entre ambos procesos? Por último, ¿ icuáles son las impresiones de otros consumidores especializados sobre la importancia de la marca? Para responder todas estas cuestiones se trabajará en dos niveles simultáneamente. En primer lugar, se discutirá teóricamente la factibilidad de la categoría de distinción en el contexto actual del mercado tecnológico. Sabiendo las dificultades inherentes que subyacen a una categoría pensada para un contexto de reproducción técnica limitado, por ejemplo el arte clásico frente a un contexto de reproductibilidad ilimitada dentro de la economía digital. En segundo lugar, se tratará a partir de la triangulación de metodologías cuanti- 
tativas y cualitativas de reflejar la especificidad de la circulación de productos Apple en Argentina. Sobre este último punto se analizan datos secundarios en términos de conectividad, provenientes de las búsquedas entre sistemas operativos iOS y Android identificado cuáles son las zonas urbanas donde predomina cada una de estas terminales tecnológicas. Por último, se trabajará sobre la construcción del imaginario de exclusividad asociado con Apple a partir del análisis de un focus group realizado a consumidores de tecnología en el marco del proyecto UBACYT "Sensibilidades e imaginarios en las producciones culturales de la Argentina en la última década, ¿hay nuevos consumos culturales?” con sede en el Instituto Gino Germani de la Universidad de Buenos Aires.

\section{La dialéctica de la distinción}

La dialéctica de la distinción (Bourdieu, 1998, 2014) constituye uno de los pilares fundamentales de la sociología de la cultura y, más específicamente, del análisis de la producción y el consumo cultural debido al enorme potencial explicativo para traducir en el plano simbólico, las desigualdades que existen materialmente en el espacio social. De esta manera, ciertas producciones artísticas, maneras de consumo y estilos de vida, que son observados y realizados en las interacciones cotidianas, se convierten en indicadores visibles del sistema de posiciones vigentes al interior de cualquier campo. No por casualidad el mundo del arte representa el universo predilecto en donde se despliegan las diferentes estrategias de pretensión y distinción, ya que al tratarse de un ámbito circunscripto con reglas de juego preestablecidas se impone tanto la singularidad irreductible del artista expresado en el culto del genio creador como la irreductibilidad de su acto de producción basado en la libertad creativa. A fin de cuentas, siempre hablando al interior del campo de la producción restringida, léase en el denominado arte serio, el valor del objeto cultural debe ser lo suficientemente maleable para poder traducir y visibilizar las diferencias.

La misma lógica se desarrolla en el campo de los bienes de lujo donde el sujeto productor y su objeto simbólico permanecen conectados entre sí por medio de una serie de propiedades que se vuelven extensivas a ambos. De ahí que los productos de la cultura material se ordenan de acuerdo a los gustos sociales señalando su distancia correspondiente al mundo de la necesidad ya que, dentro de los límites de la circulación restringida, el objeto cultural cumple siempre la función de un signo de demarcación que permite no sólo la clasificación de las personas y las cosas sino también, de gustos y actitudes personales en relación con la posición de clase, en otras palabras, de lo que se aspira a tener y lo que no. Esta cualidad del objeto cultural de funcionar como un signo de distinción depende tanto de su rareza en el mundo social como también, del carácter restringido de su proceso de circulación. Esto significa que para que el objeto cultural opere como un marcador de las condiciones sociales debe cumplir los criterios de aquellas mercancías que reciben su valor económico de la escasez (Ricardo, 1985: 22). En síntesis: deben ser un producto que existe en cantidades limitadas y donde la competencia opera con severas restricciones.

Aquí se encuentra el principal obstáculo para la aplicación de la dialéctica de la distinción al mundo de la tecnología digital. No sólo porque se trata de mercancías cuya oferta puede ser aumentada por simple voluntad humana sino también, porque el tipo de circulación 
de los dispositivos tecnológicos se asemejan mucho más al modelo de distribución masiva que al campo de la producción restringida. El problema es que en el contexto de la globalización de los mercados y de la cultura, "la inflación producida por una oferta excesiva y una rápida circulación de bienes simbólicos y de mercancías de consumo conllevan el riesgo de amenazar la legibilidad de los bienes usados como signos de status social" (Featherstone, 2000: 48). Cuando se acelera la multiplicación y circulación de los objetos tecnológicos, acaso el rasgo distintivo de toda mercancía reproducible, resulta cada vez más difícil estabilizarlos como signo de distinción de las distintas oposiciones y jerarquías culturales. Con la difusión global de la electrónica de consumo, la vorágine en la que se encuentran los dispositivos tecnológicos en el mercado, los ha vuelto mucho menos sensibles para expresar las diferencias sociales.

Ahora bien: ¿esto implica descartar la noción de distinción para el análisis del mundo de la tecnología en el sentido de considerar el modelo teórico de Bourdieu demasiado estrecho para la aleatoriedad de un mercado frenético y a gran escala? ¿Puede una mercancía de consumo masivo ser correlativa de determinadas posiciones sociales y no simplemente un efecto del gusto individual o del ingreso familiar correspondiente? ¿Hasta qué punto se puede hablar de que en el nuevo capitalismo predomina un híper consumidor individualizado (Lipovetsky, 2007), desprovisto de cualquier posicionamiento social, cuando se constituyen comunidades de consumo tan sólidas y permanente en torno a marcas tecnológicas como en el de los consumidores de Apple? Acaso no ha sido una estrategia de la empresa de Cupertino, la fidelización del cliente al instalar una lógica de la distinción en campañas publicitarias globales como Think Different (Siltanen, 2011) o en una estrategia de marketing inspirado en las marcas de lujo en lo que respecta a la importancia del packaging, la política precios al consumidor o la certificación de accesorios compatibles con la marca. De lo que se puede estar seguro es que ese conjunto de operaciones restrictivas, a primera vista discutibles desde el punto de vista comercial, ha permitido rodear a la marca Apple con un aura de exclusividad.

De ahí parece provenir ese carácter diferencial que caracteriza a los consumidores de Apple dentro de la electrónica de consumo. Frente a otras marcas tecnológicas, mucho más orientadas a una oferta híper segmentada dentro del sistema gama, lo que significa variedad de modelos para presupuestos diferentes; la estrategia comercial de Apple fue la opuesta: reducir al mínimo la cantidad de productos para concentrarse en la gama superior del mercado tecnológico y, dentro de ésta, en el segmento de consumidores que estuvieran dispuestos a pagar un plus por una mercancía de calidad diseño-intensiva. No hay que perder de vista que hasta comienzos del 2001, Apple era una marca minoritaria en términos de cuota de mercado, pero sumamente influyente al interior de las profesiones creativas vinculadas con la imagen (publicistas, diseñadores, etc.). Sin embargo, su despegue definitivo y reconfiguración posterior como gigante tecnológico va a estar ligada a la constitución de un estilo de vida transnacional. Ya sea dentro de las fracciones superiores de la clase media urbana como de los sectores ejecutivos de la burguesía transnacional los mismos principios que caracterizan a la empresa de Cupertino organizan el imaginario social de un consumo globalizado.

Llegado a este punto se debería reconsiderar la importancia de la "dialéctica de la distinción” y su aplicación pertinente a la circulación de los productos Apple en la Argentina 
durante el último trienio. No tanto porque sean considerados un objeto de "lujo" en el sentido corriente de la palabra, es decir, fundada sobre una operación de exclusión generalizada sobre el consumo de determinados bienes -nadie en su sano juicio consideraría la adquisición de un iPhone en este primer sentido de la palabra- sino mucho más al considerar a ciertos bienes como valiosos y refinados "entendiendo por refinamiento toda confección de los objetos que puede estimarse superflua para la realización de los fines necesarios" (Sombart, 1958: 88). Esta nueva definición de "lujo" mucho menos restrictiva que la anterior, parece aplicarse con mayor flexibilidad al universo de los objetos tecnológicos donde no es común que el refinamiento de la forma predomine sobre el contenido de la materia. En un mercado caracterizado por la innovación permanente, no son las funciones exóticas sino la plusvalía simbólica que produce Apple la que permite convertir a los dispositivos tecnológicos en un deleite de los sentidos.

$\mathrm{Si}$ a esto le sumamos el grado de escasez relativa con la que los productos circulan en el mercado argentino, se puede coincidir, que los productos Apple pueden operar, bajo determinadas condiciones restringidas, como un signo de distinción entre los consumidores de tecnología. Menos excluyente y jerárquico que el lujo tradicional, esta especie de consumo tecnológico suntuario permite realizar dos operaciones simultáneas. Por un lado, establecer una comunidad de consumidores vinculados a determinados marcas globales (Starbucks, Gap, etc.); por el otro, trazar una distancia simbólica con respecto a otras marcas que poseen una mayor circulación dentro de la electrónica de consumo a nivel nacional (Samsung, LG, Sony, etc.). Como si el uso de dispositivos Apple pudiera servir tanto como punto de referencia de un estilo de vida trasnacional, que en términos reales o imaginarios, se define con una norma de consumo cosmopolita y al mismo tiempo para señalar la distancia que separa con aquellos consumidores que, por efecto de las restricciones vigentes, adquieren dispositivos tecnológicos dictados por la necesidad. Ya sea de manera horizontal o vertical, el objeto tecnológico opera como marcador cultural señalando propiedades compartidas en el mapa social de la identidad.

\section{De la exclusión a la exclusividad}

El argumento central de este trabajo descansa sobre la siguiente premisa: que se puede considerar a los productos Apple en Argentina como un objeto de "lujo" en la medida en que su circulación doméstica responde a una serie de características que resultan distintivas de todo régimen suntuario. La primera de ellas es la restricción por precio o por ley a las elites; en segundo lugar, la complejidad de adquisición, la cual puede estar o no en función de la "escasez" real; en tercero, el virtuosismo semiótico, es decir la capacidad para comunicar mensajes sociales muy complejos; en cuarto, el conocimiento especializado en tanto prerrequisito de su consumo adecuado y por ultimo, un alto grado de vinculación con el cuerpo y la personalidad (Appadurai, 1991: 56). La aplicación de estas cinco características permiten entender el carácter disfuncional de la demanda (Leibenstein, 1950) de los productos Apple sino también demostrar, cómo un objeto corriente y funcional como puede ser un dispositivo tecnológico puede convertirse en un signo de distinción cuando su principal función es retórica y social. 
La primera característica se observa en los productos Apple en la Argentina es que su circulación se encuentra atravesada por una condición de escasez relativa frente a la oferta de otras marcas disponibles. Esta especificidad se debe no tanto a la inexistencia de vendedores privados o de distribuidores oficiales sino a ciertas medidas arancelarias de corte proteccionista que se establecieron desde finales del año 2009 como parte de una estrategia de promoción de la industria de ensamblaje radicada en la provincia de Tierra del Fuego. En ese entonces, durante la primera presidencia de Cristina Fernández de Kirchner, se impulsó un "impuesto tecnológico" que les permitía a los fabricantes de celulares que desearan vender en el mercado doméstico, importar libre de impuestos la totalidad de los componentes electrónicos siempre y cuando el proceso de ensamblaje se realizara en territorio nacional. De esta manera, algunas empresas líderes a escala global como es el caso de Samsung y LG optaron por ingresar al modelo de promoción industrial argentino con la consiguiente ventaja de disponer de un mercado protegido y en rápido crecimiento. Sin embargo Apple decidió rechazar la propuesta. Por diversas razones, fundamentalmente, logísticas prefirió desembarcar en la región mediante la instalación de una filial de la empresa taiwanesa Foxconn, su socio productivo en Brasil. Para ser justos, en términos estrictamente legales, el iPhone no estuvo prohibido en la Argentina, aunque las dificultades para conseguir los permisos para las licencias de importación funcionaron hasta 2017 como una prohibición de facto. Mientras que el resto del catalogo de productos de la marca (iPods, iPads, iMacs, etc.), no alcanzados por la legislación anterior, circulan hasta el día de hoy en el mercado doméstico con un sobreprecio producto de la sumatoria acumulativa de aranceles de importación (18\%), impuestos internos a los bienes considerados suntuarios (21\%) e Impuesto al Valor Agregado (21\%). Si a esto se le agrega los costos locales de comercialización y, obviamente, el margen de ganancia se llega a una relación que es mayor al doble de su costo en origen (Carrier, 2017). Basta con un simple ejemplo de la ratio del precio en la página oficial de Apple y en alguno de los retailers oficiales arroja un 2,35 para la Macbook Air, 1,9 para el último iPad y 2,5 para el iPhone 8. Con este último, Argentina obtiene el premio al "iPhone más caro del mundo" (Wasilevsky, 2017). De modo que existe un importante sustento empírico para aplicar la categoría de "escasez relativa" al consumo de Apple en Argentina. Dadas las restricciones políticas y las penalidades tributarias impuestas durante la última década han transformado a la Argentina, en el país con menor porcentaje de dispositivos móviles con iOS de toda América Latina. Según los datos de la consultora E-marketer (2015), en enero del 2015 representaba un porcentaje de hasta el $5 \%$ menos que el promedio global, ubicándose junto a Brasil en los peores puestos de América Latina. Mientras que, en el mismo año, la participación del iPhone en Chile alcanzó el $36 \%$ de los dispositivos móviles, si bien es un valor que se encuentra muy por encima del promedio global, aún así representa más del doble de la participación que en Brasil (16 \%) y más del triple de la Argentina (11\%). La rareza percibida en materia de circulación de mercancías proviene de un grado de escasez muy superior a la media con respecto a cualquier otro dispositivo tecnológico dentro del mercado nacional. Esta característica inicial, sumada a las dificultades estructurales que su adquisición supone, conduce a identificar el consumo de cualquier producto Apple como un verdadero signo de distinción. 
Esta condición del objeto tecnológico se encuentra reforzada por la modalidad de la compra imperante durante el período de restricción analizado (2015-2018). Si uno tomara por ejemplo, un año al azar, y analizara el volumen de los permisos de importación del iPhone descubriría que éstos representan una porción ínfima de las terminales tecnológicas activas en la Argentina. Se puede determinar que "entre enero del 2016 y mediados del 2017 se importaron tan sólo 17.500 teléfonos Apple mediante despachos aduaneros formales" (Wasilevsky, 2017). Sin embargo, este número resulta insignificante frente a la estimación del millón de iPhones que se encuentran en actividad. Por lo que se puede deducir que la gran mayoría de estos productos han ingresado al país por medio del turismo en el exterior y/o el mercado ilegal. De ahí que la posibilidad de acceder a un objeto distintivo en términos de circulación doméstica a un valor similar o inferior al de cualquier otra marca con presencia estable en la Argentina denota la correlación entre el turismo emisivo y la adquisición de los dispositivos móviles. Como si la compra de un iPod, un iPhone o un iPad funcionaran como un soporte identitario para la fracción superior de las clases medias metropolitanas que exhiben sus accesorios tecnológicos como pasaporte de vacaciones en el exterior.

Pero lo que resulta más interesante es que esta operación de asimilar el viejo y tradicional recuerdo fotográfico con un souvenir tecnológico lejos de verse como una especie de bluff, es decir, un atajo social para acceder a una mejora relativa en términos de status social por medio del consumo se vive como una práctica similar a la apertura de un café Starbucks en una ciudad de provincia o con los productos de la marca estadounidense Gap sin revendedores oficiales en el país, o en otro tiempo, simplemente con la compra de un bolso de viaje. Estas mercancías de marca son productos que poseen un enorme virtuosismo simbólico ya que fueron, en algún momento, productos de acceso en el sentido en que sólo podían ser adquiridas por los consumidores que viajaban al exterior. Esta condición de consumismo turístico (Roche, 2003: 65) refuerza el carácter suntuario de Apple en la Argentina en la medida en que el turista vive la experiencia del consumo como una aventura que contrasta con las obligaciones cotidianas, de modo que en este sentido el objeto tecnológico se encuentra atravesado por un determinado nivel de refinamiento que lo aleja del sustrato utilitario y de la mera necesidad.

Por eso se puede sostener que la dinámica del viaje y el mapa social de la tecnología definen las nuevas coordenadas sobre las que se construyen las nuevas formas de la identidad para amplios fracciones de los sectores medios metropolitanos. La expansión del turismo en el exterior y creciente exhibición social de la tecnología han sido fundamentales para la transformación del dispositivo tecnológico en un accesorio de moda. De aquí se desprende la cuarta característica de un régimen suntuario: la impronta utilitaria y funcional queda relegada frente a la dimensión estética que gana terreno a medida que aumenta la exposición social (Ianni \& Romani, 2018, 69) del dispositivo. Por eso el modelo de consumo distintivo operado por el ecosistema de los productos Apple se encuadra en la zona superior del esquema siguiente, el cual combina el grado de exposición del objeto tecnológico en términos de visibilidad social y el tipo de sensibilidad dominante que lo acompaña. 


\section{Eje de la visibilidad}

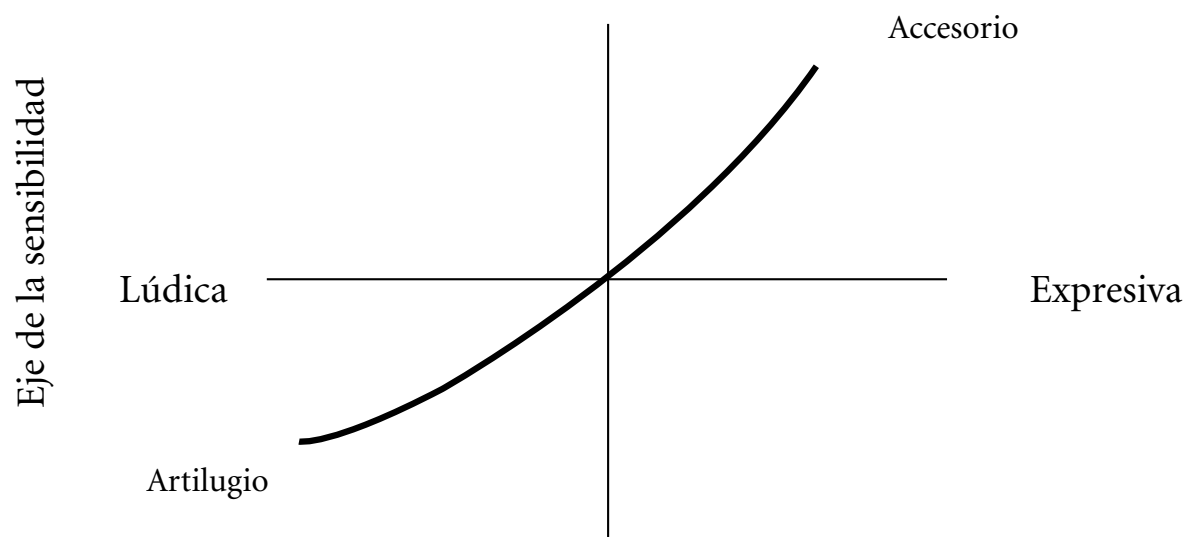

Cuadro No 1. Elaboración propia.

La zona izquierda coincide con el objeto tecnológico en cuanto gadget, es decir un dispositivo ultra funcional que satisface una utilidad sumamente específica. Pueden estar regidos por un nivel bajo de visibilidad (abajo a la izquierda) como es el caso de los denominados periféricos (mouse, trackpads, etc.) que representan una función específica pero complementaria del dispositivo principal; o por una mayor visibilidad (arriba a la izquierda) en el caso de los wearables, es decir el conjunto de los dispositivos tecnológicos con microprocesadores integrados al cuerpo por ejemplo el iWatch, los earpods, etc. Mientras que los dispositivos tecnológicos portables al estilo iPod, iPhone, iPad se alejan cada vez más del formato gadget en la medida en que están organizados por la integración funcional, menos por la lógica ultra utilitaria que por la estética mercantil. Las prácticas distintivas se realizan en la mitad superior del gráfico donde la cercanía con el cuerpo (a la izquierda) y la personalidad del consumidor (a la derecha) convierten a los dispositivos tecnológicos en signos dentro de una zona de distinción.

De aquí se desprende la quinta característica distintiva del modelo suntuario, la cual define la correlación que existe entre los atributos de la marca y la personalidad del consumidor. Como ninguna otra compañía del sector, la empresa de Cupertino ha apostado a mantener un control absoluto sobre el proceso de producción y distribución, lo que implica una planificación minuciosa que va desde la concepción del diseño, la elección de los materiales hasta la forma en la que se presentan los productos en los respectivos puntos de venta. La adición del prefijo "i” como elemento distintivo funciona no sólo como un signo de identificación de la marca dentro de la electrónica de consumo -se sabe que un iPhone 
es un teléfono de Apple- pero también un iPad es una tableta que se conecta directamente a internet por lo que el prefijo "i” opera como un significante vacío que busca extender los atributos simbólicos de la mercancía en cuestión: la innovación, la imaginación y la inteligencia a la personalidad del consumidor. El éxito de esta operación permite desarrollar una lógica distintiva por medio del consumo, mucho menos asociado con el efecto Veblen que con la demanda snob.

\section{(No) Tan distintos}

A lo largo de este trabajo se han planteado varias cuestiones sobre la relación entre tecnología y consumo. En primer lugar, la posibilidad de aplicar la categoría de distinción a la electrónica de consumo sabiendo las dificultades inherentes a un mercado competitivo y a una mercancía técnicamente reproducible. Por ello se ha buscado definir de una manera mucho más amplia la categoría de "lujo" con el fin de poder aplicarlo al consumo de Apple en la Argentina. Aun a sabiendas, que existe una clara intencionalidad por parte de las súper marcas (Klein, 2005) en crear una imagen exclusiva se ha demostrado que, esta circunstancia nunca garantizada, fue producida artificialmente bajo determinadas condiciones excepcionales de escasez dentro del mercado nacional. La misma situación que ha llevado ha identificar la marca Apple con ciertos valores asociados a la libertad individual, a la integración internacional, al modelo liberal de la globalización, etc. Paradójicamente, la marca del Think Different se encuentra asociada en la Argentina con la defensa de los valores occidentales en el contexto de la globalización, que han despertado la circulación de determinados rumores sobre la apertura de un Apple Store (2017) en la avenida 9 de Julio de la Ciudad Autónoma de Buenos Aires coincidente con la asunción del gobierno de Mauricio Macri.

Este último apartado buscará reconstruir las bases de este imaginario social a partir de las representaciones que poseen los consumidores de Apple por medio de la información extraída de un grupo focal. El mismo permitió ahondar en las percepciones y miradas de los consumidores en pos de la construcción y reproducción de estrategias de distinción y diferenciación por medio del sistema de marcas. Esto servirá para comprender cómo ciertas formas de consumo son re significadas en un contexto complejo, cargado de información y saturación (Bhaskar, 2017), en el cual las elecciones, deseos y aspiraciones sociales se ven inmersas en un escenario dominado por el exceso de oferta y en permanente actualización. En el caso argentino, se le suman a lo anterior, las condiciones excepcionales que imponen un modelo de circulación restringida, lo cual ha significado que algunos elementos del imaginario Apple aparezcan potenciados, frente a otros que pasan a un segundo plano. Resulta una obviedad repetir que el éxito de Apple se basa en la importancia que se le atribuye al diseño, algo que la empresa viene privilegiando desde siempre: los ordenadores y sus programas coinciden en algunos aspectos con los del entorno Microsoft o Android; sin embargo, la firma ha sabido concebir un mundo Apple, en el que el ordenador, por sus líneas, su sistema de navegación y su grafismo define un estilo de vida e induce a pertenecer a un grupo selecto que comparte sus valores: "los industriales, los banqueros, los comerciantes son Microsoft; los editores, los publicistas, los intelectuales, los jóvenes, la gente a 
la última [moda] son Apple" (Lipovetsky \& Serroy, 2015: 194). No hay marca sin diseño creativo: aquella se identifica gracias a éste y por éste se diferencia de sus competidores en el mercado. En una época en que los productos alcanzan de manera creciente el mismo nivel técnico, hay que encontrar la forma de salir del montón, atraer la mirada mediante "algo más" que permita diferenciarlos.

¿Quién usa Apple? El que prioriza diseño, interfaz, accesibilidad, interacción con el aparato y no le importa por ahí el precio, y el resto de las marcas, por ahí Samsung, le interesa un buen teléfono que funcione, pero por ahí no tiene para acceder a algo mejor (Valentín, 27 años - Consultor free lance).

Esa diferencia o plusvalía simbólica que existe en el plano del objeto mercantil puede desarrollarse de manera horizontal o vertical en la medida en que sirva para describir un conjunto de propiedades compartidas al interior de un grupo social o para diferenciar entre distintas fracciones de clase. Si en el primer caso, la distinción que se opera es horizontal o intraclase por ejemplo entre profesiones creativas y liberales; en el segundo se traduce en una operación de diferenciación vertical o interclase entre quienes pueden acceder a los dispositivos Apple en el país y los que no. Por eso se puede hablar que la marca de la manzana se encuentra en la Argentina mucho más identificada con la segunda forma de distinción que con la primera en tanto responde a un target social de las fracciones superiores de las clases medias y altas. Un nicho específico dentro de un mercado masivo, lo cual se contrapone con uno de los pilares básicos del diseño moderno: que es la de concebir un producto de vanguardia al alcance de todos.

Este rasgo distintivo de los dispositivos Apple se puede corroborar a partir de un estudio realizado por una consultora inmobiliaria (Properati, 2018) en el área metropolitana de Buenos Aires (AMBA) con la búsqueda de propiedades por zonas y rangos de precios entre las terminales Android e iOS. Con un material de más de 15000 mensajes durante tres meses se contrasta la presencia de las formas de distinción horizontal y vertical. La primera, se observa en la brecha que existe entre usuarios Android e iOS se reduce en aquellos barrios con mayor presencia de la denominada clase creativa (Florida, 2010). Por ejemplo, en la zona de Parque Patricios donde se ubica el Distrito Tecnológico Metropolitano, la proporción entre los usuarios de las distintas plataformas se encuentra casi igualado. Lo que resulta paradigmático teniendo en cuenta la escasez relativa de los dispositivos Apple en la ciudad. Mientras que, en términos de distribución geográfica, se refuerza el carácter social de los mismos en la medida en que las búsquedas de "los usuarios de iOS se concentran en la zona norte del AMBA -mucho más cara en términos de U\$/m2 de la ciudad- $y$ contactan a los inmuebles más costosos que los propios usuarios de Android.

De modo un entrevistado expresa lo que siente al usar un dispositivo Apple como "sentirse parte de una elite". No tanto por las características que poseen los productos ni por el precio pagado por ellos en el mercado doméstico -se ha con anterioridad señalado que la mayoría de los productos son comprados en el exterior o ingresan al país de manera ilegal- sino, fundamentalmente, por el sentido de exclusividad que se encuentra adherido a ellos. 
Igual en el mundo de Apple lo que pasa es que no lo podés comprar acá, tenés que ir y comprarlo afuera. Si lo comprás acá en un local, que es oficial digamos, te saldría un tanto por ciento más de lo que sale afuera, con impuestos, todo (Marcelo - Programador).

Este carácter exclusivo de Apple en Argentina habilita un modo singular de vincularse con los dispositivos tecnológicos portátiles puesto que la marca aparece como sinónimo de excelencia, con una supremacía marcada con respecto a las demás empresas del sector. El mismo individuo continúa diciendo:

Yo trataba de explicarle a una persona que me preguntaba por qué Apple era así, y analizando todas las marcas, las demás marcas tratan de crecer en el mercado, que más gente consuma sus marcas y sus productos, y Apple lo que trata de hacer es que los mismos que lo usan reciban algo mejor.

En palabras del entrevistado, la estrategia de ventas de la empresa californiana parece contrastar con la política comercial de otros gigantes del mundo high tech. La diferencia radica en que el criterio de expansión del volumen de ventas, o en el sentido de un aumento de la cuota de mercado, no se realiza por medio de una estrategia basada en la reducción agresiva del precio o un crecimiento invasivo de la publicidad sino por medio de una mejora constante en la calidad del producto. Lo cualitativo se impone aquí frente a lo cuantitativo, como un sello determinante de la marca símbolo del consumo global. No por casualidad, con este testimonio se puede develar que la estrategia de Apple difiere radicalmente de la lógica del "lujo tradicional" mucho más ligada a la escasez absoluta de los materiales o a la producción limitada por encargo que a un mercado de circulación masiva a escala global. Por el contrario, el ecosistema de productos Apple describe un comportamiento singular en Argentina donde la demanda potencial aumenta a medida que disminuye (relativamente) la cantidad imaginaria de consumidores. Como si el valor simbólico de la rareté del dispositivo tecnológico justificara el precio excedente que se está dispuesto a pagar para acceder a la marca. Por eso los consumidores de Apple en Argentina pagan por mercancías minoritarias al interior de un mercado porque de allí deriva su excedente simbólico que se traduce en una forma de status social. Al igual que lo que sucede en otros mercados de status, como los consumidores de vinilo, en los coleccionistas de relojes, etc. se observa un caso de disfuncionalidad de la demanda en el sentido del efecto snob (Leibenstein, 1950). Lo que significa que la demanda puede aumentar, no tanto porque el precio del bien sea elevado sino porque la extensión de su consumo está fuertemente limitada en un determinado ámbito. Frente al vaciamiento simbólico de las mercancías que produce la globalización, son pocas las que pueden funcionar como soportes materiales de una nueva identidad social. Detrás del aura de exclusividad que envuelve a los productos Apple en Argentina emerge para el consumidor doméstico como un nuevo signo de distinción. 


\section{Bibliografía}

(2015). “Argentina, el país con menos usuarios de iPhone de la región.”. Infobae. Recuperado de https://www.infobae.com/2015/04/27/1725074-argentina-el-pais-menos-usuariosiphone-la-region/.

(2017). "Crecen los rumores sobre la apertura de una tienda oficial Apple en Argentina" Clarín. Recuperado de https://www.clarin.com/sociedad/crecen-rumores-aperturatienda-oficial-apple-argentina_0_H1sIa7Kae.html.

Appadurai, A. (1991). La vida social de las cosas. México: Grijalbo.

Bhaskar, M. (2017). Curaduría. El poder de la selección en un mundo de excesos. México: FCE.

Bourdieu, P. (1998). La distinción. Criterio y bases sociales del gusto. Madrid: Taurus.

Bourdieu, P. (2014). El sentido social del gusto. Elementos para una sociología de la cultura. Buenos Aires: Siglo XXI.

Carrier, E. (2017). “La manzana (ya no) prohibida.” Recuperado de http://www.comentarios blog.com.ar/2017/03/31/la-manzana-ya-no-prohibida/.

Featherstone, M. (2000). Cultura de consumo y posmodernismo. Buenos Aires: Amorrortu.

Florida, R. (2010). La clase creativa. La transformación de la cultura del trabajo y el ocio en el siglo XXI. Barcelona: Paidós.

Ianni, M. y Romani, M. (2018). "El consumidor tecnológico: la emergencia de una nueva sensibilidad en una economía de la experiencia." En Un mundo de sensaciones. Sensibilidades e imaginarios en producciones y consumos culturales argentinos del siglo XXI. A. Wortman. Buenos Aires: CLACSO. 57-81 pp.

Klein, N. (2005). No Logo. El poder de las marcas. Buenos Aires: Paidós.

Leibenstein, H. (1950). "Bandwagon, Snob and Veblen Effects in the Theory of Consumers' Demand”. The Quaterly Journal of Economics 64 (2): 183-207 pp.

Lipovetsky, G. (2007). La felicidad paradójica. Ensayo sobre la sociedad de hiperconsumo. Barcelona: Anagrama.

Lipovetsky, G. y Serroy, J. (2015). La estetización del mundo. Buenos Aires: Anagrama.

Properati (2018). “Android vs iOS.” Recuperado de http://blog.properati.com.ar/androidvs-ios/ 2017.

Ricardo, D. (1985). Principios de Economía política y tributación (selección). Madrid: Orbis.

Roche, M. (2003). Mega-events and Modernity. Olympics and expos in the growth of global culture. New York: Routledge.

Siltanen, R. (2011). The real story behind Apple's 'Think Different' campaign. Forbes.

Sombart, W. (1958). Lujo y Capitalismo. Buenos Aires: Guillermo Dávalos editor.

Wasilevsky, J. (2017). "Índice iPhone 8: qué puesto ocupa Argentina en el ranking mundial de precios del celular de Apple." iProfesional.

Abstract: The present work intends to discuss the scope of the category of "distinction" and its problematic application to the world of digital technology. For this, it takes as a universe of analysis the Apple consumers in Argentina during the last three years (2015- 
2018), in order to understand the impact that imposed a restricted circulation of certain goods on the corporate values that characterize the brand. The hypothesis that is tried to demonstrate is that as of the 2010 -when the policies in the matter of importation of technological goods hardened- that the Apple's consumer experience began to be perceived like a strategy of distinction among the Argentine consumers.

Keywords: Distinction - Tecnological devices - Consumer experiences - Material culture - New luxury - Social status - Snobbery.

Resumo: O presente trabalho pretende discutir o alcance da categoria de "distinção" e sua aplicação problemática ao mundo da tecnologia digital. Para isso, toma-se como universo de análise os consumidores da Apple na Argentina durante os últimos três anos (20152018), a fim de compreender o impacto que impunha uma circulação restrita de certos bens nos valores corporativos que caracterizam a marca. A hipótese que se tenta demonstrar é que a partir de 2010 -quando as políticas em matéria de importação de bens tecnológicos se endureceram- que a experiência de consumo da Apple começou a ser percebida como uma estratégia de distinção entre os consumidores argentinos.

Palavras chave: Distinção Dispositivos tecnológicos - Experiências de consumo - Cultura material - Novo luxo - Status social - Esnobismo.

[Las traducciones de los abstracts fueron supervisadas por el autor de cada artículo] 